\title{
Magnetically aligned dust and SiO maser polarisation in the envelope of the red supergiant VY Canis Majoris
}

\author{
W. H. T. Vlemmings ${ }^{1}$, T. Khouri ${ }^{1}$, I. Martí-Vidal ${ }^{1}$, D. Tafoya ${ }^{1}$, A. Baudry ${ }^{2}$, S. Etoka ${ }^{3}$, E. M. L. Humphreys ${ }^{4}$, \\ T. J. Jones ${ }^{5}$, A. Kemball ${ }^{6}$, E. O'Gorman ${ }^{7}$, A. F. Pérez-Sánchez ${ }^{8}$, and A. M. S. Richards ${ }^{9}$ \\ 1 Department of Earth and Space Sciences, Chalmers University of Technology, Onsala Space Observatory, 43992 Onsala, Sweden \\ e-mail: wouter.vlemmings@chalmers.se \\ 2 Laboratoire d'astrophysique de Bordeaux, Univ. Bordeaux, CNRS, B18N, allée Geoffroy Saint-Hilaire, 33615 Pessac, France \\ 3 Hamburger Sternwarte, Gojenbergsweg 112, 21029 Hamburg, Germany \\ ${ }^{4}$ European Southern Observatory, Karl-Schwarzschild-Str. 2, 85748 Garching, Germany \\ 5 Minnesota Institute for Astrophysics, University of Minnesota, Minneapolis, MN 55455, USA \\ 6 Department of Astronomy and National Center for Supercomputing Applications, University of Illinois at Urbana-Champaign, \\ 1002 W. Green Street, Urbana, IL 61801, USA \\ 7 Dublin Institute for Advanced Studies, 31 Fitzwilliam Place, Dublin 2, Ireland \\ 8 European Southern Observatory, Alonso de Córdova 3107, Vitacura, Casilla 19001, Santiago, Chile \\ 9 Jodrell Bank Centre for Astrophysics, School of Physics and Astronomy, University of Manchester, Manchester M13 9PL, UK
}

Received 6 March 2017 / Accepted 11 April 2017

\begin{abstract}
Aims. Polarisation observations of circumstellar dust and molecular (thermal and maser) lines provide unique information about dust properties and magnetic fields in circumstellar envelopes of evolved stars.

Methods. We use Atacama Large Millimeter/submillimeter Array (ALMA) Band 5 science verification observations of the red supergiant VY CMa to study the polarisation of $\mathrm{SiO}$ thermal/maser lines and dust continuum at $\sim 1.7 \mathrm{~mm}$ wavelength. We analyse both linear and circular polarisation and derive the magnetic field strength and structure, assuming the polarisation of the lines originates from the Zeeman effect, and that of the dust originates from aligned dust grains. We also discuss other effects that could give rise to the observed polarisation.

Results. We detect, for the first time, significant polarisation $(\sim 3 \%)$ of the circumstellar dust emission at millimeter wavelengths. The polarisation is uniform with an electric vector position angle of $\sim 8^{\circ}$. Varying levels of linear polarisation are detected for the $J=4-3{ }^{28} \mathrm{SiO} v=0,1,2$, and ${ }^{29} \mathrm{SiO} v=0,1$ lines, with the strongest polarisation fraction of $\sim 30 \%$ found for the ${ }^{29} \mathrm{SiO} v=1$ maser. The linear polarisation vectors rotate with velocity, consistent with earlier observations. We also find significant (up to $\sim 1 \%$ ) circular polarisation in several lines, consistent with previous measurements. We conclude that the detection is robust against calibration and regular instrumental errors, although we cannot yet fully rule out non-standard instrumental effects.

Conclusions. Emission from magnetically aligned grains is the most likely origin of the observed continuum polarisation. This implies that the dust is embedded in a magnetic field $>13 \mathrm{mG}$. The maser line polarisation traces the magnetic field structure. The magnetic field in the gas and dust is consistent with an approximately toroidal field configuration, but only higher angular resolution observations will be able to reveal more detailed field structure. If the circular polarisation is due to Zeeman splitting, it indicates a magnetic field strength of $\sim 1-3$ Gauss, consistent with previous maser observations.
\end{abstract}

Key words. supergiants - stars: mass-loss - stars: magnetic field - polarization - masers - stars: individual: VY CMa

\section{Introduction}

The study of the stellar winds of evolved massive stars is important not only because the amount of expelled mass is a key factor in determining the subsequent evolution of the star, and its eventual fate, but also because they have a profound impact on the interstellar medium and evolution of the Galaxy via their material and energy output. However, the mechanisms that drive mass loss from these massive stars are not well understood. It is generally assumed that the acceleration of the stellar wind in low- to intermediate-mass asymptotic giant branch (AGB) stars is mainly due to radiation pressure on dust grains (e.g. Habing 1996; Höfner 2008, and references therein). However, the formation of dust grains that are non-transparent to stellar radiation is inhibited in the vicinities of evolved massive stars due to their extreme luminosity (Speck et al. 2000; Woitke 2006; Norris et al. 2012). It is thus puzzling how the material is transported from the surface of the star to the radius where non-transparent dust species form. Furthermore, it has been observed that the distribution of material in the circumstellar envelope (CSE) of red supergiant stars is far from spherical (e.g. Humphreys et al. 2007; O'Gorman et al. 2015). This suggests the action of an aspherical mechanism in the acceleration of the gas. While several models have been proposed to explain the (anisotropic) mass loss in evolved massive stars (including convection, pulsation, scattering off large grains, acoustic and magnetic waves; e.g. Hartmann \& MacGregor 1980; Josselin \& Plez 2007; Höfner 2008), it remains unclear which of these mechanisms plays the dominant role.

Spectroscopic imaging of the outflows in evolved stars gives important clues about their geometry and velocity fields, thus the physics that governs them. In addition, polarimetric observations of the molecular and continuum emission provide 
Table 1. Spectral window setup.

\begin{tabular}{|c|c|c|c|c|c|c|}
\hline Spw & $\begin{array}{c}\text { Centre frequency } \\
{[\mathrm{GHz}]}\end{array}$ & $\begin{array}{c}\text { Channel width } \\
{[\mathrm{MHz}]}\end{array}$ & {$\left[\mathrm{km} \mathrm{s}^{-1}\right]$} & Number of channels & $\begin{array}{c}\text { Total bandwidth } \\
{[\mathrm{GHz}]}\end{array}$ & Line(s) \\
\hline 0 & 183.310 & 0.244 & 0.40 & 960 & 0.2344 & para- $\mathrm{H}_{2} \mathrm{O} 3_{13}-2_{20}$ \\
\hline 1 & 183.884 & 1.953 & 3.18 & 960 & 1.8750 & - \\
\hline 2 & 170.999 & 1.953 & 3.42 & 960 & 1.8750 & $\begin{array}{l}{ }^{28} \mathrm{SiO}(J=4-3, v=2) \\
{ }^{29} \mathrm{SiO}(J=4-3, v=0) \\
{ }^{29} \mathrm{SiO}(J=4-3, v=1)\end{array}$ \\
\hline 3 & 172.481 & 0.244 & 0.42 & 480 & 0.1172 & ${ }^{28} \mathrm{SiO}(J=4-3, v=1)$ \\
\hline 4 & 173.668 & 0.244 & 0.42 & 480 & 0.1172 & ${ }^{28} \mathrm{SiO}(J=4-3, v=0)$ \\
\hline
\end{tabular}

valuable information on the magnetic field and characteristics of the dust grains. Specifically, polarimetric continuum observations can reveal the common orientation of elongated dust grains around evolved stars due to the effect of a global magnetic field in a similar way as has been observed in star forming regions (e.g. Cortes et al. 2016), and planetary nebulae (e.g. Sabin et al. 2014). Observations of polarised maser emission, from molecules such as $\mathrm{SiO}, \mathrm{H}_{2} \mathrm{O}$, and $\mathrm{OH}$, have also been important in the determination of the strength and geometry of the magnetic field in the expanding envelopes of evolved stars (e.g. Kemball \& Diamond 1997; Vlemmings et al. 2005; Etoka \& Diamond 2010). This type of observation can be used to probe the magnetic field in the CSE of evolved massive stars and determine its relation to the mass loss phenomenon.

An excellent example of a massive evolved star with a high mass-loss rate $\left(\sim 10^{-4} M_{\odot} \mathrm{yr}^{-1}\right)$ is VY CMa (Danchi et al. 1994; Decin et al. 2006; Humphreys et al. 2007). The high dust content in its CSE makes it an attractive target to explore the processes of mass loss and dust formation in massive stars (Richards et al. 2014; De Beck et al. 2015; O'Gorman et al. 2015). It is also one of the largest red supergiant stars, located at a distance of $1.2 \mathrm{kpc}$ (Zhang et al. 2012). Recent high angular resolution ALMA Band 7 (around $321 \mathrm{GHz}$ ) and 9 (around $658 \mathrm{GHz}$ ) observations, tracing scales down to 60 mas, revealed the presence of two enigmatic features detected only in continuum emission at submillimetre wavelengths. The brightest of them, dubbed "clump C", is located 334 mas south-east of VY CMa, while the other structure, VY, is located toward the north, elongated in the direction perpendicular to the line that connects the star and clump C (O'Gorman et al. 2015, hereafter OG+15). It is unlikely that these structures formed from convection cells since this would require a constant mass loss in a single direction for a few decades, but it has been suggested that they could have been formed as a result of localised long-lived magnetohydrodynamic disturbances in the photosphere $(\mathrm{OG}+15)$. From polarisation observations of the maser emission, the strength of the magnetic field in the CSE was found to range from a few $\mathrm{mG}$ to a few G (Vlemmings et al. 2002; Herpin et al. 2006; Richter et al. 2016). Such observations however, do not clearly reveal the global structure of the magnetic field within the CSE. In this paper we present the analysis of ALMA full polarisation Band 5 science verification observations towards the star VY CMa and report the detection of polarised line and continuum emission around the star that is used to probe the magnetic field and determine its association with features seen in its CSE.

\section{Observations and data reduction}

These observations are part of the ALMA Band 5 (163$211 \mathrm{GHz}$ ) Science Verification (SV) campaign. The VY CMa observations took place on 16 October 2016, starting at 05:45 UT and lasted for about $6.4 \mathrm{~h}$. A total of 13 antennas were used in the calibration and imaging. The observations were performed using five spectral windows (spws). The spw setup is presented in Table 1.

The full calibration and imaging was performed in CASA 4.7.0, and is described in the Band 5 polarisation calibration memo (Martí-Vidal et al. 2016) that was released with the data. The gain calibration (bandpass, amplitude, absolute fluxdensity scale and phase) was performed following the standard ALMA procedures. The amplitude and phase gains for spw 0 (i.e. the window centred at $183.299 \mathrm{GHz}$ ), which has the narrowest bandwidth $(234.4 \mathrm{MHz})$, were derived by transferring the gain solutions from spw 1 (total bandwidth of $1.875 \mathrm{GHz}$ ).

The polarisation calibration was also performed following standard ALMA procedures, although with some improvements that we summarise here. The $X-Y$ cross-polarisation phases at the reference antenna were fitted by pre-averaging spw 0 and spw 1 in chunks of 200 and 20 channels, respectively. This was done to increase the signal-to-noise ratio of the gain solutions close to the atmospheric water-vapour line. Also, the calibration tables were created on a per-spw basis. This was needed to properly derive the ambiguities of the $X-Y$ phases (see Martí-Vidal et al. 2016, Sect. 4.2, for more details).

Finally, we performed four iterations of self-calibration on a strong channel of the $\mathrm{SiO}$ maser in spw 3 . These solutions were applied to all the other spw. We then reimaged all the spws at full spectral resolution and both the continuum and lines at full polarisation, producing data cubes in Stokes $I, Q, U$, and $V$. The imaging of the spectral lines was performed using Briggs weighting with a robustness parameter of 0.5 , which resulted in a synthesised beam of $\sim 0.8 \times 0.3^{\prime \prime}$. The typical $\mathrm{rms}$ noise in line free channels around the $\mathrm{SiO}$ lines was $\sim 3.8 \mathrm{mJy} \mathrm{beam}^{-1}$ for spw 3, and 4 , and $\sim 1.4 \mathrm{mJy}$ beam $^{-1}$ for the coarser spectral resolution of spw 2. The continuum was imaged using a robust gridding weighting parameter of -0.5 resulting in a beam of $\sim 0.5 \times 0.2^{\prime \prime}$. We achieved an rms noise of $0.1 \mathrm{mJy}^{\text {beam }}{ }^{-1}$.

According to the calibration procedure described in Martí-Vidal et al. (2016) and used here, the estimates of the instrumental polarisation in the spws close to the atmospheric water line (i.e. spw 0 and spw 1) may be biased due to the higher noise in the water-vapour frequency window and/or to the mapping function (i.e. dependence with antenna elevation) of the atmospheric absorption. The polarisation calibration in these spws should thus be taken with care. With respect to spw 2-4, the polarisation calibration is not affected by such a bias. Therefore, we use only the linear-polarisation results from spw 2-4 (covering the $\mathrm{SiO}$ masers and the continuum) in our analysis. Regarding the images in circular polarisation (i.e. Stokes $V$ ), a Stokes $V$ signal can be affected by defects or biases in the calibration of 

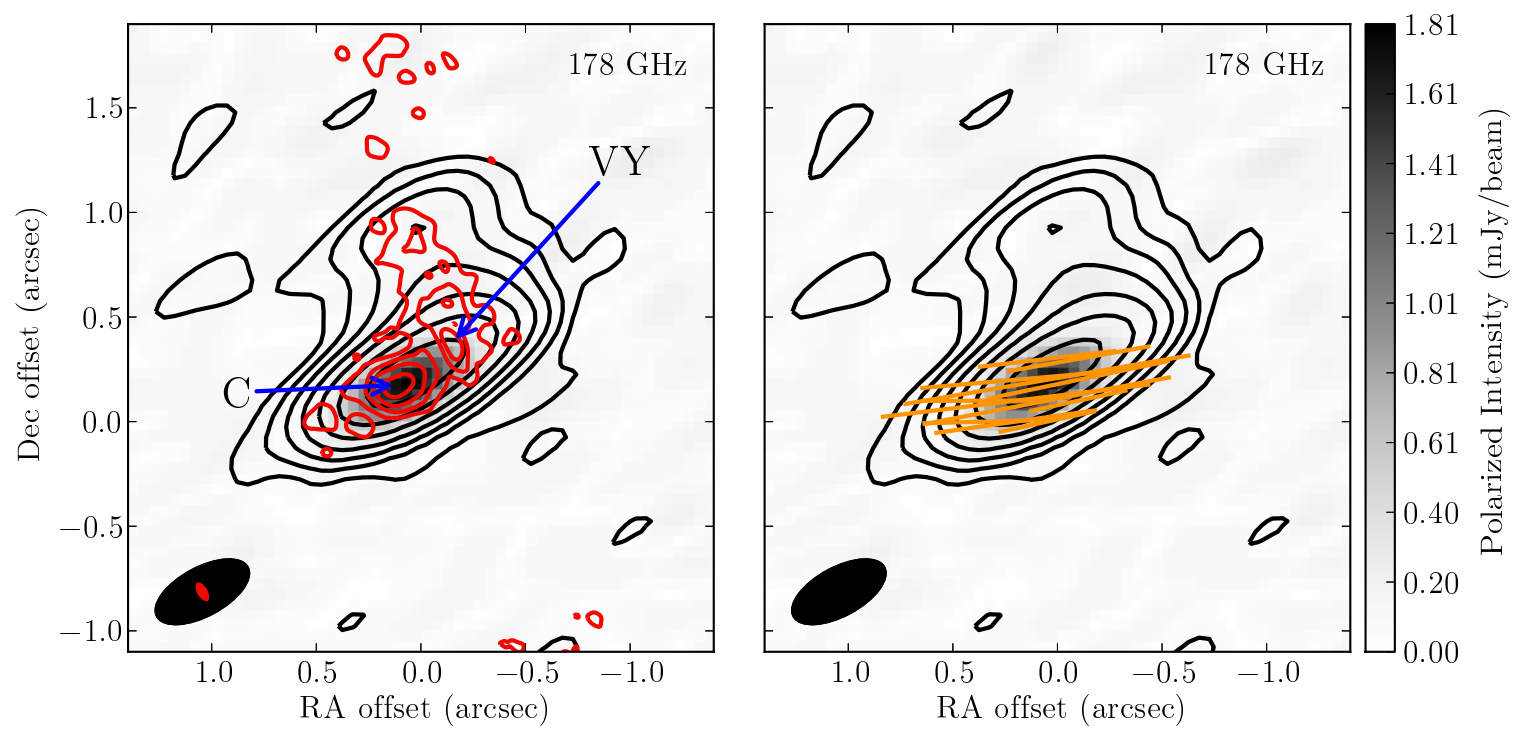

Fig. 1. The dust at $178 \mathrm{GHz}$ around of VY CMa. Arrows indicate dust clump C and the star (VY) identified in previous ALMA observations (Richards et al. 2014, OG+15). The grey-scale image is the linearly polarised intensity, which is seen to peak at the bright dust component clump C in the South-East. The black contours are the total intensity at $0.01,0.02,0.04,0.08,0.16,0.32$, and 0.64 times the peak brightness. The similarly spaced red contours (left) indicate the ALMA $658 \mathrm{GHz}$ continuum from OG+15. The vectors (right) indicate the direction of polarisation rotated by $90^{\circ}$ to indicate the magnetic field direction if the polarised emission originates from magnetically aligned grains. The beam size is indicated in the lower left corner.

the instrumental polarisation; from a biased estimate of the phase between the polarisers at the reference antenna to biased (and correlated) estimates of the polarisation leakage at all the antennas. A justification of the fidelity of these Stokes $V$ images, together with a robustness test of the instrumental polarisation calibration of these data, is described in Appendix A). Based on this analysis, we rule out calibration errors or standard instrumental effects as the origin of the observed circular polarisation. However, as these are the first reported ALMA circular polarisation results, there is still the chance that they are affected by unkown instrumental issues.

\section{Results}

\subsection{Dust continuum}

In order to map the continuum, we only used line-free channels sufficiently far from the atmospheric $\mathrm{H}_{2} \mathrm{O}$ line. The resulting aggregate bandwidth was $2.25 \mathrm{GHz}$. The VY CMa continuum, centred around $178 \mathrm{GHz}$, is extended. Although the beam is elongated exactly along the direction between component $\mathrm{VY}$ and the continuum clump $\mathrm{C}$ seen in $(\mathrm{OG}+15)$, the distribution appears identical when the ALMA Band 7 and Band 9 observations $(\mathrm{OG}+15)$ are convolved with the Band 5 beam. In Fig. 1 (left) we show the Band 5 continuum overplotted on the ALMA Band 9 observations around $658 \mathrm{GHz}$. Deconvolving the two components, we measure a total flux of $36 \pm 3 \mathrm{mJy}$ for the star and $94 \pm 9 \mathrm{mJy}$ for clump C. These values are completely consistent with the average spectral index of the two components determined from the Band $7(321 \mathrm{GHz})$ and Band $9(658 \mathrm{GHz})$ observations $(\mathrm{OG}+15)$. This implies that clump $\mathrm{C}$ is still optically thick at $178 \mathrm{GHz}$ and increases, following the analysis from $\mathrm{OG}+15$, the dust mass to $>1.2 \times 10^{-3} M_{\odot}$ and decreases the dust temperature to $\sim 100 \mathrm{~K}$. As in $\mathrm{OG}+15$, we did not detect the SW clump seen in Hubble observations of scattered light and a number of molecular lines (e.g. Humphreys et al. 2007; De Beck et al. 2015).
We also detect clear linear polarisation. The maximum fractional polarisation is $\sim 3 \%$. The polarised intensity is indicated in grey-scale in Fig. 1 and clearly peaks at the location of clump C. Despite the large beam, there is no polarisation found associated with VY or the North-East dust extension, even though the sensitivity would have been sufficient to detect both down to $1-2 \%$. In Fig. 1 (right) we show the polarisation vectors rotated by $90^{\circ}$ to reflect the magnetic field direction if the polarisation is due to the emission from magnetically aligned grains. The average (nonrotated) electric vector position angle (EVPA) is $8 \pm 4^{\circ}$ (East of North), where the error includes both the scatter around the average and the error due to the noise in the polarisation images. The vectors are thus neither tangential nor parallel to the direction of the star to clump $\mathrm{C}$, which has a position angle of $\sim 130^{\circ}$.

\subsection{SiO emission}

The observational setup included several lines of $\mathrm{SiO}$ and its ${ }^{29} \mathrm{SiO}$ isotope. They display maser emission in the vibrationally excited states and predominantly thermal emission in the $v=0$ state. These $\mathrm{SiO}$ maser and thermal emissions are associated with the VY component towards the star, and not with clump C. The line strength and polarisation of the $\mathrm{SiO}$ lines covered are listed in Table 2 along with their transition energy and Einstein $A$ coefficients (Müller et al. 2001). In the rest of the paper we omit $J=4-3$ when we describe the transitions. All the maser lines show a complicated line structure, but are not, or only marginally, spatially resolved in our $0.8 \times 0.3^{\prime \prime}$ beam. Their spectra, in Stokes I, linear polarisation fraction, EVPA, and Stokes $V$, are shown in Figs. 2-4. The strongest linear polarisation, with a fraction up to $\sim 30 \%$, is detected for the ${ }^{29} \mathrm{SiO}$ $v=1$ transition with polarisation up to $\sim 20 \%$ detected for ${ }^{28} \mathrm{SiO}$ $v=2$. With the exception of a single, possibly saturated maser feature (as discussed in Sect. 4.1), the polarisation of the ${ }^{28} \mathrm{SiO}$ $v=1$ line is around 5\%. The linear polarisation fraction is consistent with estimates based on recent APEX observations of the same transitions (Humphreys et al. 2017). In Fig. 5, we present 
Table 2. Observed SiO lines.

\begin{tabular}{llccccc}
\hline \hline $\begin{array}{l}\text { Rest frequency } \\
{[\mathrm{GHz}]}\end{array}$ & Transition & $\begin{array}{c}E_{\mathrm{u}} / k \\
{[\mathrm{~K}]}\end{array}$ & $\begin{array}{c}A \\
{\left[\mathrm{~s}^{-1}\right]}\end{array}$ & $\begin{array}{c}\text { Peak flux } \\
{[\mathrm{Jy}]}\end{array}$ & $\begin{array}{c}P_{1, \max } \\
{[\%]}\end{array}$ & $\begin{array}{c}\left|P_{\mathrm{c}, \max }\right| \\
{[\%]}\end{array}$ \\
\hline \multicolumn{7}{c}{${ }^{28} \mathrm{SiO}$} \\
\hline $173.688^{a}$ & $J=4-3, v=0$ & 21 & $2.602 \times 10^{-4}$ & 2.4 & 4.0 & 1.4 \\
$172.481^{a}$ & $J=4-3, v=1$ & 1790 & $2.569 \times 10^{-4}$ & 104.2 & 20.1 & 0.8 \\
$171.275^{b}$ & $J=4-3, v=2$ & 3542 & $2.547 \times 10^{-4}$ & 69.4 & 18.5 & -1.1 \\
\hline \multicolumn{7}{c}{${ }^{29} \mathrm{SiO}$} \\
\hline $171.512^{b}$ & $J=4-3, v=0$ & 21 & $2.505 \times 10^{-4}$ & 3.2 & 2.0 & $<0.3$ \\
$170.328^{b}$ & $J=4-3, v=1$ & 1779 & $2.484 \times 10^{-4}$ & 7.4 & 28.6 & 0.9 \\
\hline
\end{tabular}

Notes. ${ }^{(a)}$ Observed at $0.42 \mathrm{~km} \mathrm{~s}^{-1}$ spectral resolution. ${ }^{(b)}$ Observed at $3.4 \mathrm{~km} \mathrm{~s}^{-1}$ spectral resolution.
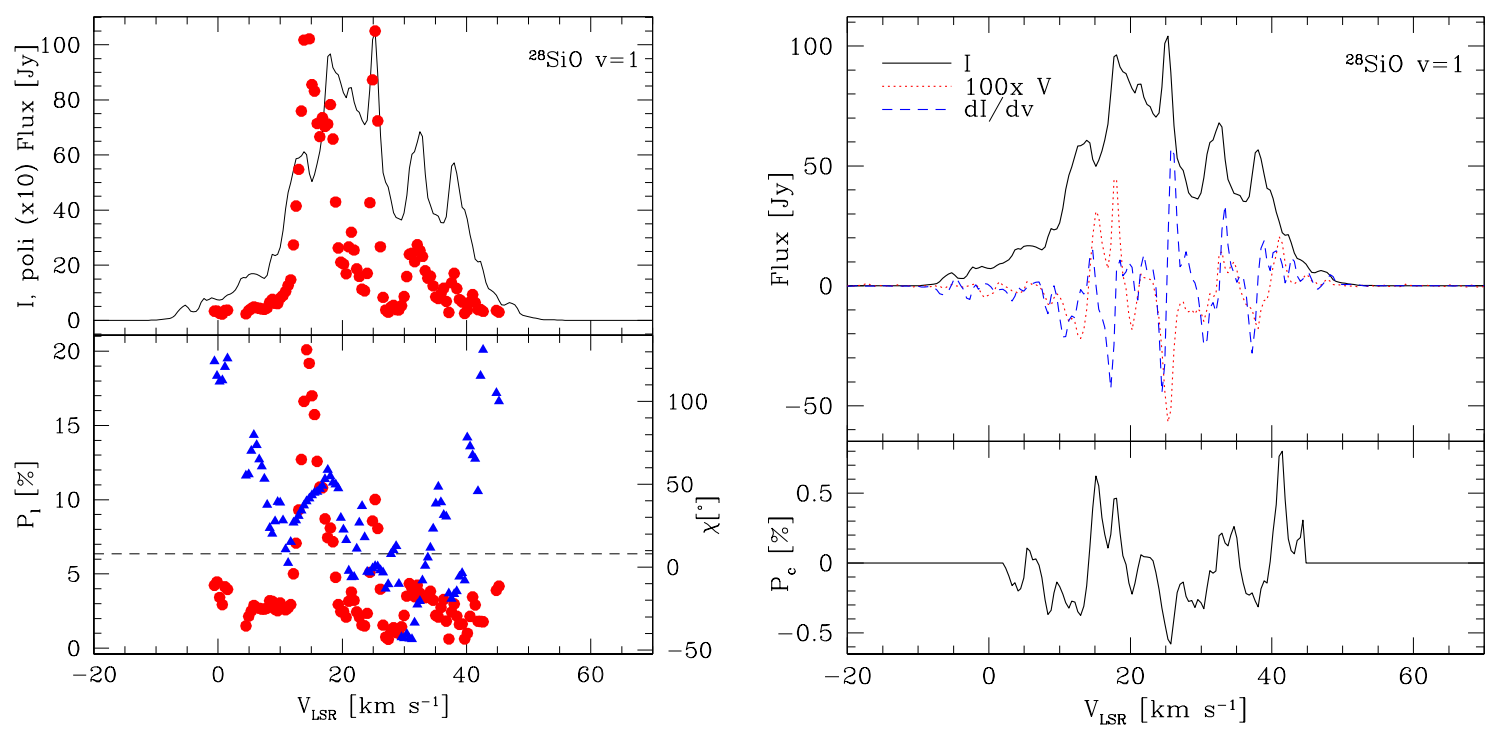

Fig. 2. Left, top: Total intensity (solid line) and linearly polarised intensity (solid red circles) from the ${ }^{28} \mathrm{SiO}(J=4-3, v=1)$ transition. The linear polarisation spectrum is multiplied by a factor of 10. Left, bottom: The linear polarisation fraction (red dots) and electric vector position angle (EVPA) (blue triangles). The horizontal dashed line indicates the EVPA observed in the dust continuum. Right, top: The circularly polarised emission (red short-dashed, multiplied by 100$)$ for the ${ }^{28} \mathrm{SiO}(J=4-3, v=1)$ transition. Overplotted is also the total intensity derivative $\mathrm{d} I / \mathrm{d} v$ (blue long-dashed) scaled for a magnetic field strength of $\sim 2.4 \mathrm{G}$. Right, bottom: The circular polarisation fraction $P_{\mathrm{c}}$, which is found to peak at $\sim 0.8 \%$ for this transition.
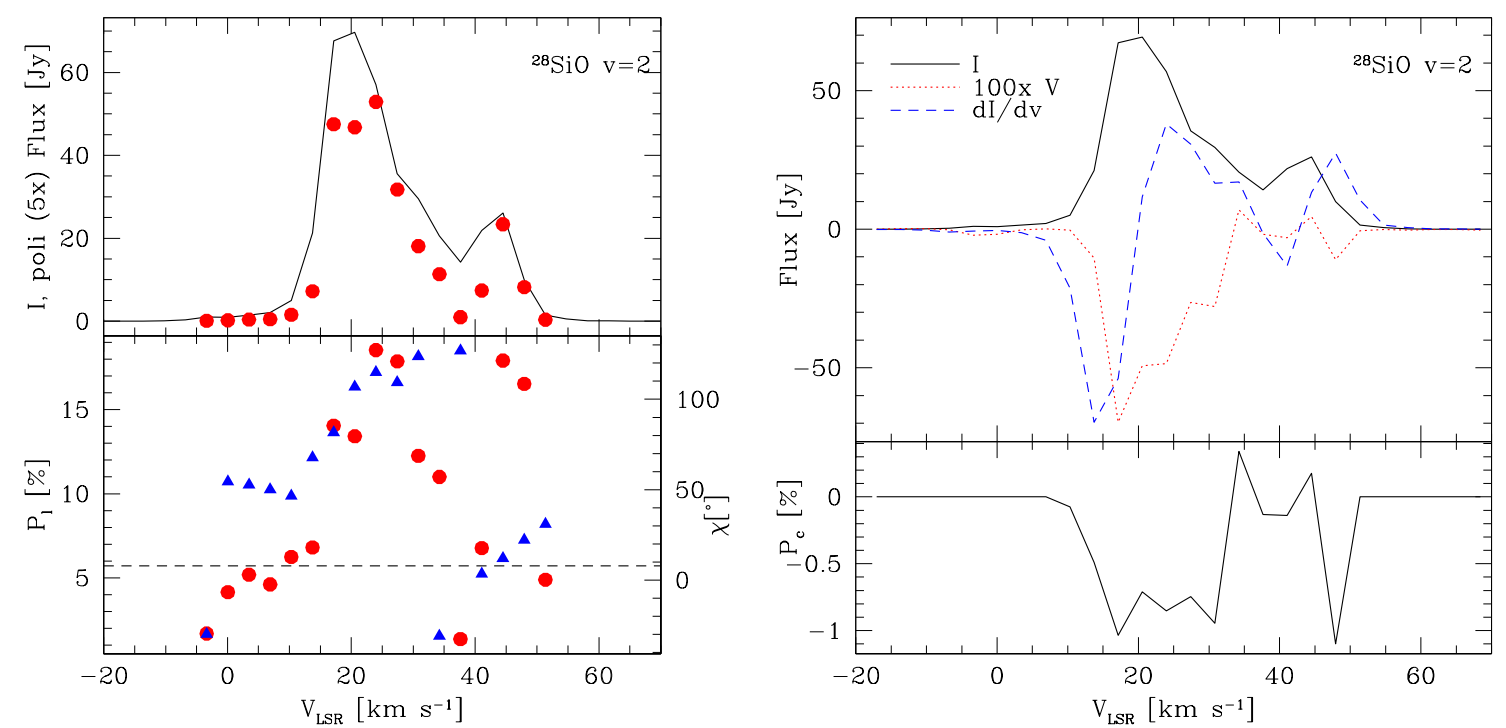

Fig. 3. As Fig. 2 for the ${ }^{28} \mathrm{SiO}(J=4-3, v=2)$ transition. The linear polarisation spectrum is multiplied by five. 
W. H. T. Vlemmings et al.: Magnetically aligned dust and $\mathrm{SiO}$ maser polarisation around VY CMa
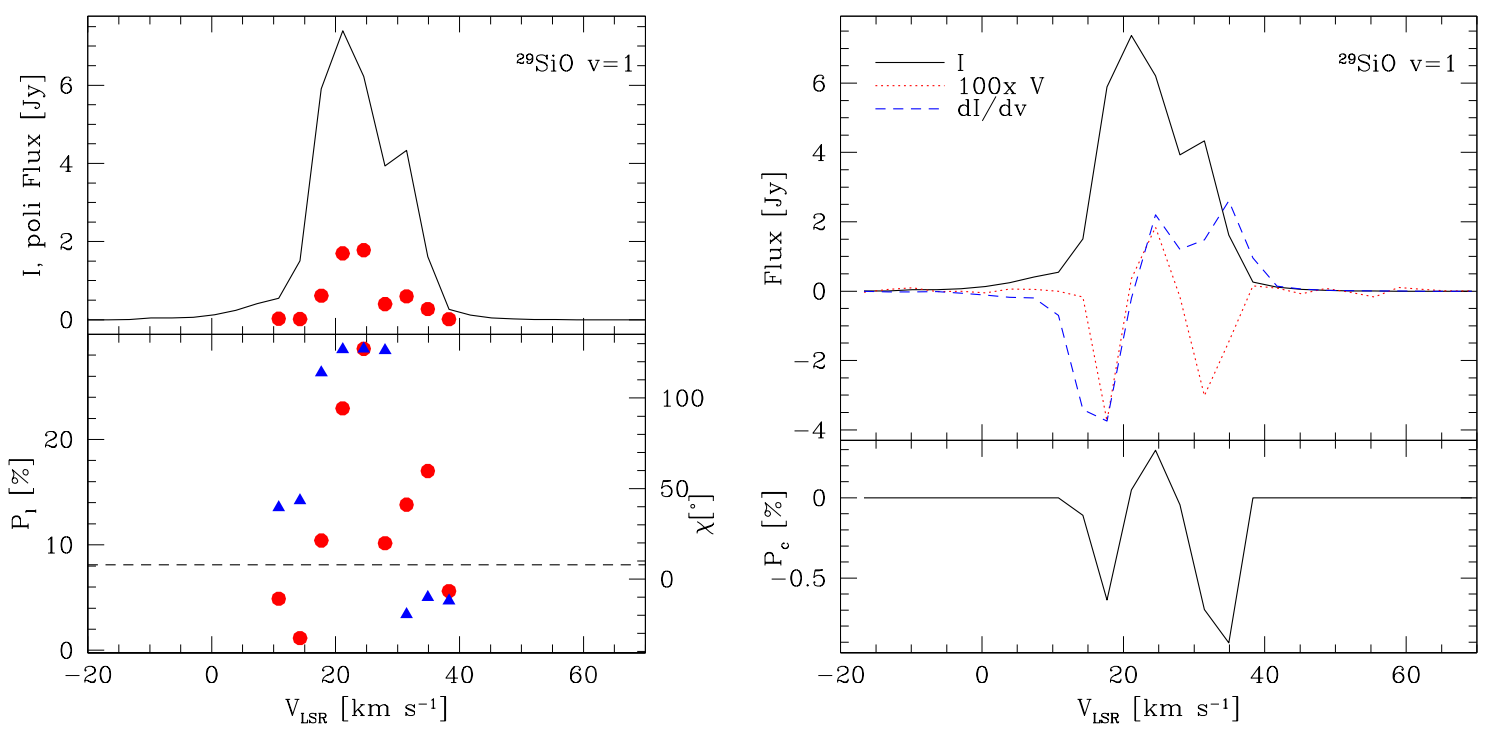

Fig. 4. As Fig. 2 for the ${ }^{29} \mathrm{SiO}(J=4-3, v=1)$ transition. The linear polarisation spectrum is not multiplied.

a number of channel maps of the linear polarisation for this transition. For all maser lines we also detect circular polarisation at a level up to $\sim 1 \%$. The spectral resolution for all but the ${ }^{28} \mathrm{SiO} v=1$ line is not sufficient for a more detailed analysis. Hence, unless otherwise noted, the rest of our analysis is based on the ${ }^{28} \mathrm{SiO} v=1$ transition.

The thermal $\mathrm{SiO} v=0$ line is significantly spatially extended, both in Stokes $I$ as well as in linear polarisation. The polarisation spectrum in Fig. 6, extracted in an aperture encompassing the extended polarised emission, shows two distinct spectral features. For illustration, two channel maps representing the two components, blue-shifted at $V_{\mathrm{LSR}}=21 \mathrm{~km} \mathrm{~s}^{-1}$ and red-shifted at $V_{\mathrm{LSR}}=48 \mathrm{~km} \mathrm{~s}^{-1}$, are shown in Fig. 7. The neighbouring channels show a similar structure. The polarisation pattern at $V_{\mathrm{LSR}}=21 \mathrm{~km} \mathrm{~s}^{-1}$ shows that the fractional polarisation peaks away from VY and that the polarisation vectors point radially towards the star. However, at $V_{\mathrm{LSR}}=48 \mathrm{~km} \mathrm{~s}^{-1}$, the polarisation peaks at the stellar position and does not show the radial pattern. The two linearly polarised components are also seen in the circular polarisation spectrum, which shows no sign of a typical S-shaped Zeeman pattern.

\section{Discussion}

\subsection{SiO maser polarisation}

In the framework of basic maser theory, it is important to investigate the rate of stimulated emission of the maser $R$, the maser decay rate $\Gamma$ and the Zeeman frequency $g \Omega$, in order to determine if the $\mathrm{SiO}$ maser polarisation is the result of Zeeman or non-Zeeman properties (Pérez-Sánchez \& Vlemmings 2013, and references therein). If $g \Omega>R$, and the maser is not strongly saturated $(R \lesssim 10 \Gamma)$, the magnetic field determines the linear and circular polarisation of the maser. Under those conditions, the maximum fractional linear polarisation for the $\mathrm{SiO}$ transitions we observed will be of the order of $15 \%$, depending on the angle $\theta$ between the maser propagation direction and the magnetic field. However, $\mathrm{SiO}$ masers often display higher levels of fractional polarisation. This is likely due to anisotropic pumping of the energy levels involved in the maser (e.g. Nedoluha \& Watson 1990). However, even in that case, the relationships between
$R, \Gamma$, and $g \Omega$ still determine whether the magnetic field can be traced by the maser polarisation (Watson 2002).

Using the parameters from Pérez-Sánchez \& Vlemmings (2013), $g \Omega \sim 150 \mathrm{~s}^{-1}$ for the magnetic field strength of $\sim 0.1 \mathrm{G}$ in the $\mathrm{H}_{2} \mathrm{O}$ maser region (Vlemmings et al. 2002). The radiative decay rate $\Gamma$ can be approximated by $\Gamma=5 \mathrm{~s}^{-1}$, which corresponds to the rate for the radiative decay from the first vibrationally excited state to the ground-vibrational state (Nedoluha \& Watson 1990). The stimulated emission rate $R$ depends on the maser brightness temperature $T_{\mathrm{b}}$, beaming angle $\Delta \Omega$, Einstein $A$ coefficient and transition frequency $v$ through:

$R \sim \frac{A k T_{\mathrm{b}} \Delta \Omega}{4 \pi h v}$

We adopt a value of $\Delta \Omega=10^{-2}$ sr. The brightness temperature itself depends on the size of the maser emission region. Based on VLBI observations (e.g. Richter et al. 2016), we adopt a typical maser spot size of $\sim 1$ mas. As VLBI observations generally do not recover all maser flux observed with larger angular resolution, this size corresponds to the core of the maser emission and likely results in an overestimate of $T_{\mathrm{b}}$. From this, we find that $T_{\mathrm{b}}<2 \times 10^{9} \mathrm{~K}$ for the ${ }^{28} \mathrm{SiO}$ and $T_{\mathrm{b}}<1 \times 10^{8} \mathrm{~K}$ for the ${ }^{29} \mathrm{SiO}$ masers. For the stimulated emission rate, we thus find $R<50 \mathrm{~s}^{-1}$ for ${ }^{28} \mathrm{SiO}$, and $R<8 \mathrm{~s}^{-1}$ for ${ }^{29} \mathrm{SiO}$. Thus, while the strongest of the ${ }^{28} \mathrm{SiO}$ masers could be approaching saturation, $g \Omega>R$ and $g \Omega>\Gamma$. We thus expect the magnetic field to determine the polarisation characteristics of the majority of the maser features. This agrees well with the observed fractional linear polarisation, which is mostly $<15 \%$. Only one of the strongest maser features of the ${ }^{28} \mathrm{SiO} v=1$ transition reaches a polarisation fraction of $\sim 20 \%$, which could imply that this feature is strongly saturated. This feature, around $V_{\mathrm{lsr}}=15 \mathrm{~km} \mathrm{~s}^{-1}$ also displays a change in polarisation vector that could be the result of saturation.

As shown in Fig. 2 (right), the observations of the ${ }^{28} \mathrm{SiO}$ $v=1$ also reveal significant circular polarisation. Possible spurious circular polarisation is discussed in Appendix A. Assuming a general Zeeman splitting origin, the circular polarisation spectrum $V$ is expected to be proportional to the derivative of the total intensity $I$, with a proportionality coefficient that depends on the magnetic field strength. This assumes a constant magnetic field strength with uniform direction throughout the maser. 

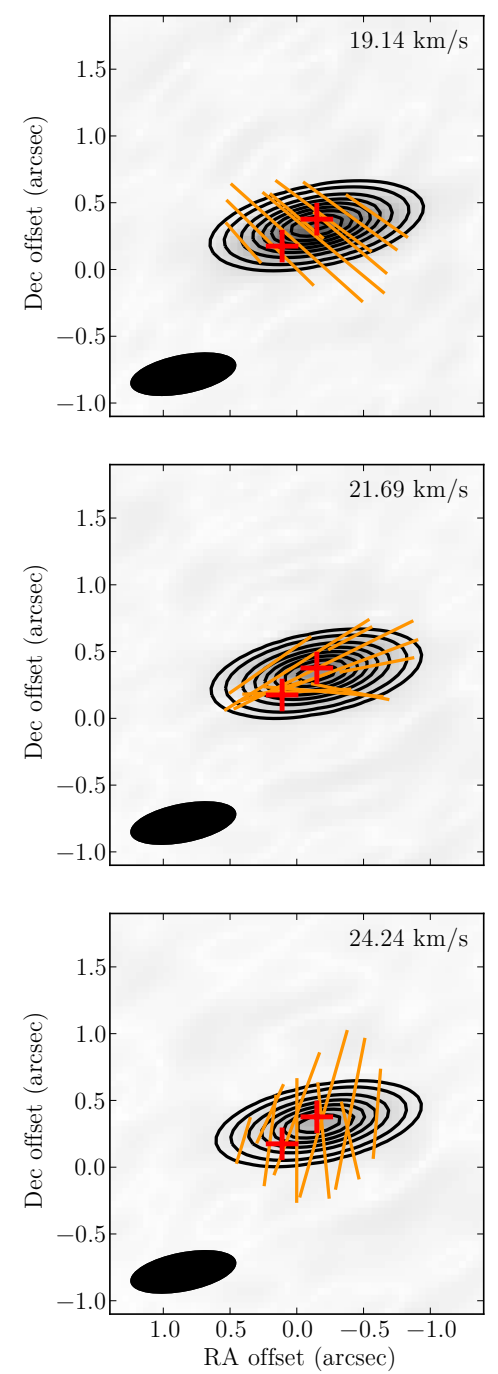
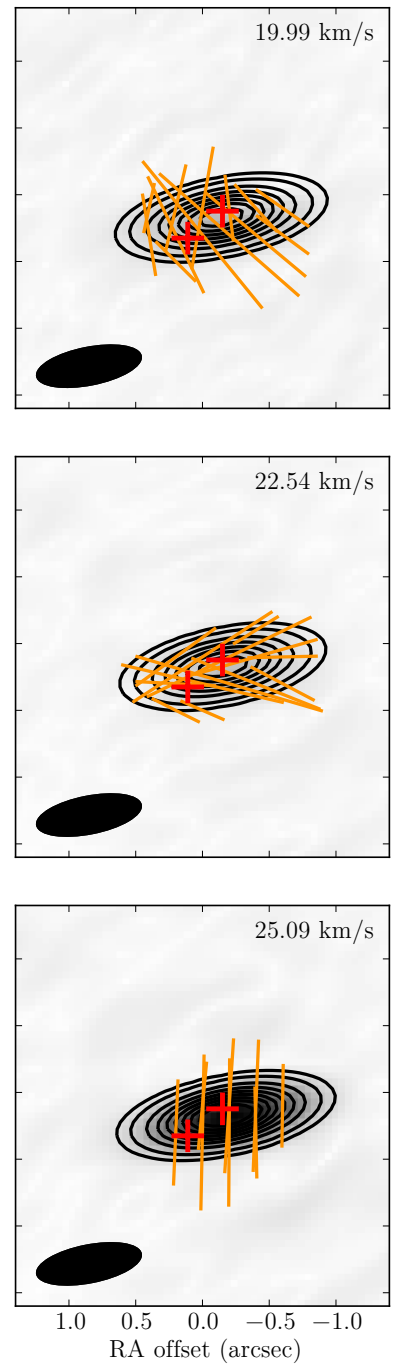
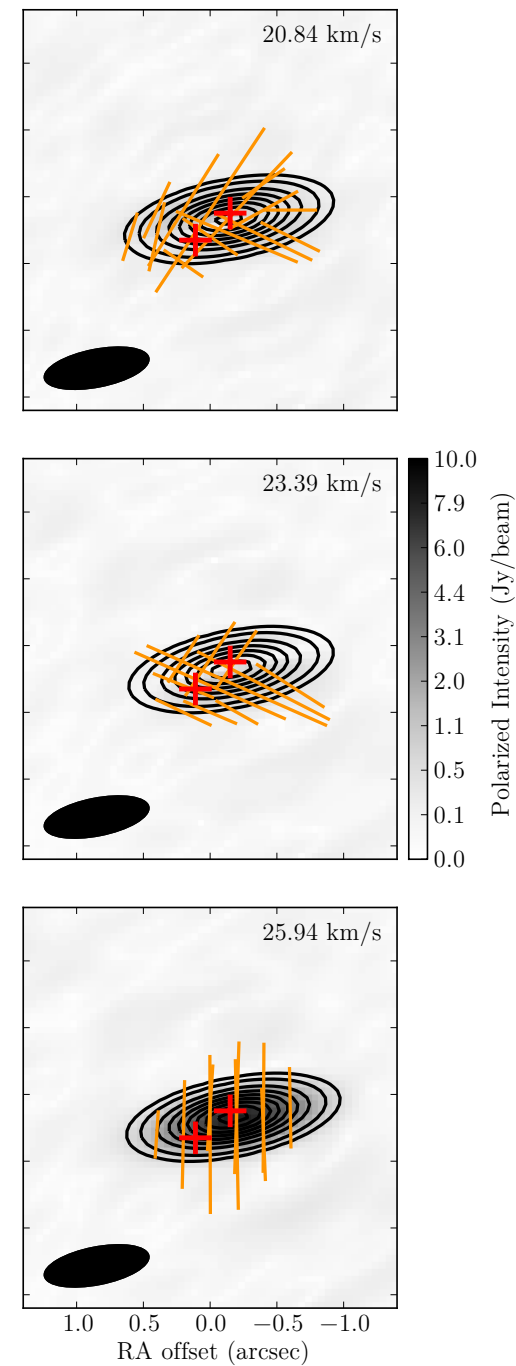

Fig. 5. Channel maps of the linear polarisation of the ${ }^{28} \mathrm{SiO}(J=4-3, v=1)$ maser at selected channels around the stellar velocity $\left(V_{\mathrm{LSR}}=\right.$ $22 \mathrm{~km} \mathrm{~s}^{-1}$ ). The (red) crosses indicate the position of the star (close to the centre of the image) and clump C (towards the south-east). The greyscale image is the linearly polarised intensity. The contours are the total intensity at $0.05,0.1,0.2,0.3,0.4,0.5,0.6,0.7$, and 0.8 times the peak flux. The vectors indicate the direction of polarisation. The beam size is indicated in the lower left corner.

As indicated by the linear polarisation, such a uniform structure is unlikely. Still, we show that $V \propto \mathrm{d} I / \mathrm{d} v$ is a reasonable approximation of the observed spectrum if one assumes a magnetic field reversal at $V_{\mathrm{lsr}} \sim 15$ and $30 \mathrm{~km} \mathrm{~s}^{-1}$, which is close to symmetric around the stellar velocity $\left(V_{\mathrm{lsr}} \sim 22 \mathrm{~km} \mathrm{~s}^{-1}\right)$. This strongly supports the basic Zeeman interpretation of the circular polarisation. Although the spectral resolution was significantly worse, circular polarisation was also detected for the ${ }^{28} \mathrm{SiO} v=2$ and the ${ }^{29} \mathrm{SiO} v=1$ maser. Also in this case $V \propto \mathrm{d} I / \mathrm{d} v$ is a good approximation. In order to obtain an estimate of the magnetic field strength required to produce the observed circular polarisation, we use the fractional circular polarisation (following Herpin et al. 2006; Kemball \& Diamond 1997; Elitzur 1996). For the $J=4-3$ masers, we find $B \approx 1.3 P_{\mathrm{c}} \cos \theta$ assuming a typical maser linewidth of $\sim 1 \mathrm{~km} \mathrm{~s}^{-1}$. We thus find $B / \cos \theta \approx$ $0.85 \mathrm{G}$. This is similar to the field strengths of $1-2 \mathrm{G}$ found using the same method from previous $J=1-0$ and $J=2-1 \mathrm{SiO}$ maser observations (Herpin et al. 2006; Richter et al. 2016), supporting the Zeeman interpretation of the observed circular polarisation. The above approximation assumes saturated masers (Elitzur 1996) and slightly different results are obtained when applying the basic Zeeman fitting that might be more applicable to the non-saturated masers we observe. We thus also determine the field strength using

$V=z B \cos \theta \frac{\mathrm{d} I}{\mathrm{~d} v}$.

Here, the proportionality term $z=g \Omega \approx 1500 \mathrm{~s}^{-1}$ [Gauss] $^{-1}$. Our fit shown in Fig. 2 (right) then yields a magnetic field strength of $B \cos \theta \sim 2.4 \mathrm{G}$. Thus, in either method, the magnetic field is indeed strong enough for $g \Omega>R$. Both field strength estimates are easily sufficient to magnetically align the dust grains in clump $C$ on a sufficiently short timescale (as described in Sect. 4.3).

Alternatively, Houde (2014) suggests the $\mathrm{SiO}$ maser circular polarisation could arise from resonant scattering of magnetised foreground $\mathrm{SiO}$ gas. This causes linear polarisation to be converted to circular polarisation. The effect has been able to explain the circular polarisation of $43 \mathrm{GHz} \mathrm{SiO}$ masers. The conversion phenomenon depends on the excitation of the molecular transition in the foreground, and the much lower column density of ${ }^{29} \mathrm{SiO}$ would lead to a conversion efficiency lower than that of ${ }^{28} \mathrm{SiO}$. As we observe a similar circular polarisation fraction, it appears that the resonant scattering does not contribute significantly to the maser transitions presented here. 
W. H. T. Vlemmings et al.: Magnetically aligned dust and SiO maser polarisation around VY CMa
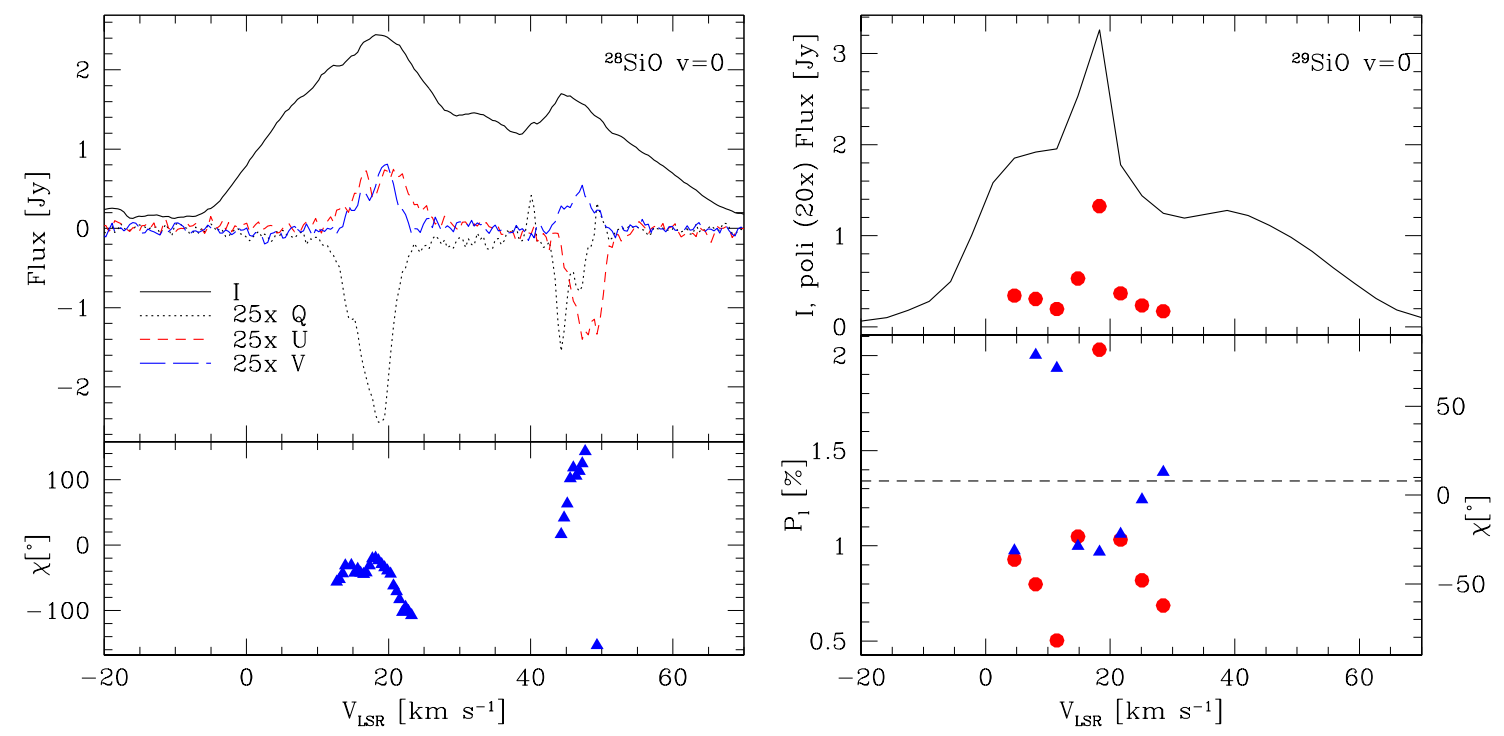

Fig. 6. Left: The Stokes $I, Q, U$, and $V$ spectra for the ${ }^{28} \mathrm{SiO}(J=4-3, v=0)$ transition extracted in the region where linear polarisation was detected. The bottom panel displays the EVPA (blue triangles) determined from the Q, and U spectra. Right: As Fig. $2(l e f t)$ for the ${ }^{29} \mathrm{SiO}(J=4-3$, $v=0$ ) transition. The linear polarisation spectrum is multiplied by 20.
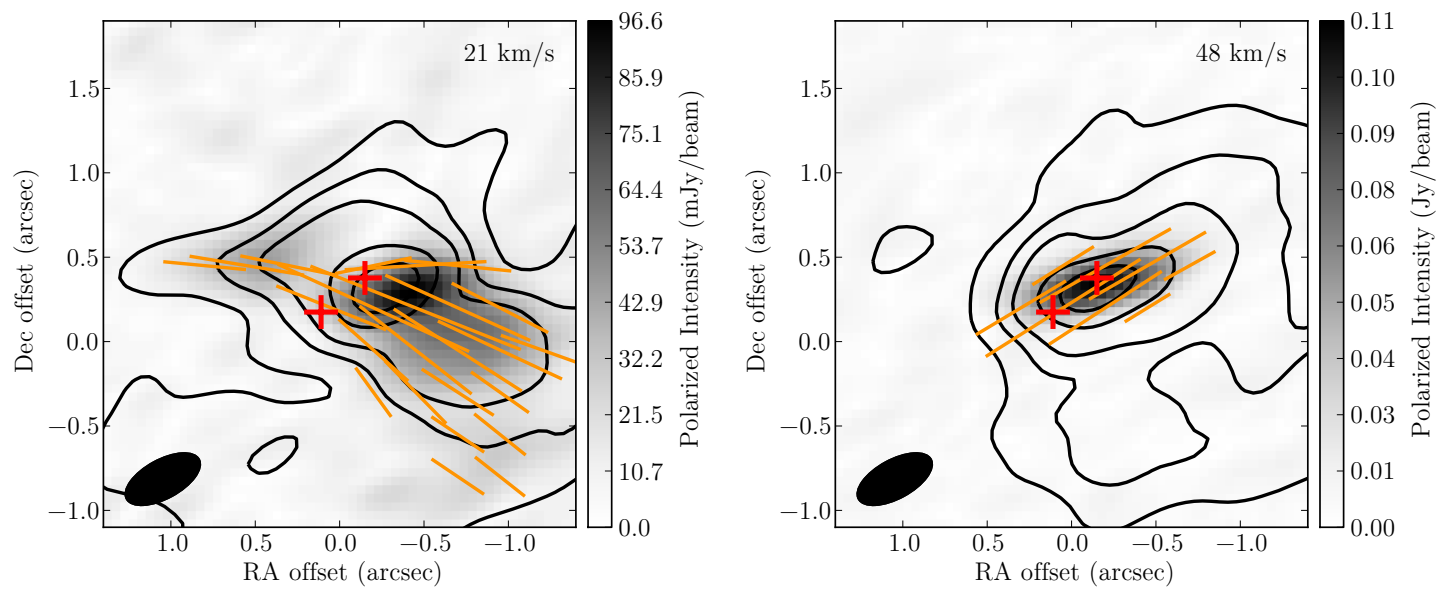

Fig. 7. Channel images of the thermal ${ }^{28} \mathrm{SiO}(J=4-3, v=0)$ polarisation of the two polarized components seen in Fig. 6 . The crosses, grey-scale, contours and vectors are as Fig. 5 with contours at $0.2,0.3,0.4,0.6$, and 0.8 times the peak.

\subsection{Thermal SiO polarisation}

Linearly polarised line emission from non-maser molecular lines can arise due to differences in excitation of the magnetic sublevels within a transition. This can occur already in a weak magnetic field when the magnetic sublevels of a rotational state experience anisotropic emission. This effect is generally called the Goldreich-Kylafis effect (hereafter GK-effect) and is described in Goldreich \& Kylafis $(1981,1982)$. Alternatively, linear polarisation in a CSE can occur from molecules with a preferred rotation axis because of strong radial infrared emission from a central star (Morris et al. 1985). Significantly polarised (thermal) molecular line emission around evolved stars has so far been observed for $\mathrm{SiO} v=0$ and $\mathrm{CO}$ in the envelope of the M-type AGB star IK Tau (Vlemmings et al. 2012) and for CS, SiS, and CO around the C-type AGB star IRC+10216 (Glenn et al. 1997; Girart et al. 2012). In the case of radiative molecular alignment in the absence of a magnetic field, the linear polarisation should mainly be perpendicular to the radial direction. Already for a magnetic field strength of a few $\mu \mathrm{G}$ however, the molecular alignment in much of the CSE is determined by magnetic field (Morris et al. 1985). In the case of the GK-effect, the linear polarisation is along the magnetic field as long as the Zeeman splitting dominates the collisional and spontaneous emission rates (Kylafis 1983).

The behaviour of thermal line polarisation will thus change depending on optical depth, distance from the star, and magnetic field strength and geometry. Our observations seem to indicate such a change, as shown in Fig. 6 (right) and Fig. 7. The polarisation is confined to a velocity range between $V_{\mathrm{lsr}}=11$ and $25 \mathrm{~km} \mathrm{~s}^{-1}$ close to and slightly blue-shifted from the stellar velocity, and a clearly redshifted component between $V_{\mathrm{lsr}}=42$ and $52 \mathrm{~km} \mathrm{~s}^{-1}$. While the (slightly) blue-shifted component displays a radial polarisation pattern that could indicate that the radiation field dominates the molecular alignment, the red-shifted component is neither radial nor tangential. The red-shifted polarisation component also peaks towards the star, contrary to predictions of radiative alignment. It thus appears that the red-shifted component traces the magnetic field according to the GK effect. This implies a magnetic field position angle of $\sim 40^{\circ}$ in the most 
red-shifted part of the CSE which is consistent with the extreme velocity $\mathrm{SiO}$ masers.

We also detect significant circular polarisation in the ${ }^{28} \mathrm{SiO}$ $v=0$ thermal line. As seen in Fig. 6 (left), the circular polarisation spectrum is significantly different from that observed for the maser lines; it displays similarities to the linear polarisation (Stokes $Q$, and $U$ ) spectra, although it does not show the negatives seen in those and it peaks at approximately $1.6 \%$ but does not show a typical Zeeman spectrum. In Appendix A, we rule out calibration errors, although unknown instrumental effects in the ALMA system can only be ruled out once the observations are repeated. Non-Zeeman circular polarisation has previously been reported for $\mathrm{CO}$ and has been suggested to be due to the resonant scattering of foreground molecules via the same mechanism suggested for $\mathrm{SiO}$ masers (Houde 2014). The excitation conditions of the thermal $\mathrm{SiO}$ foreground gas can explain why the effect is seen in the $v=0$ transition and not the maser transitions. The lower abundance of ${ }^{29} \mathrm{SiO}$ is therefore the reason why no circular polarisation was detected for the ${ }^{29} \mathrm{SiO} v=0$ line despite the total intensity being of equal strength to the ${ }^{28} \mathrm{SiO} v=0$ line.

Alternatively the velocity ranges displaying polarised emission could be those including maser components. In this case, the circular polarisation of ${ }^{28} \mathrm{SiO} v=0$ may instead arise from weakly saturated maser features. This can only be confirmed with future, higher-angular-resolution observations.

\subsection{Dust polarisation of clump C}

We consider two possible scenarios to explain the observed polarized radiation in the continuum emission towards clump $\mathrm{C}$, scattering off large dust particles or emission from non-spherical grains with a preferred orientation direction. In our analysis, we also include that observations of polarised visible light obtained with Hubble (Jones et al. 2007) and SPHERE/VLT (Scicluna et al. 2015) show that the polarisation vectors seen towards clump $\mathrm{C}$ in visible wavelengths are roughly perpendicular to those observed at $\mathrm{mm}$ wavelength presented here.

For the polarisation signal at $\mathrm{mm}$ wavelengths to be produced by scattering off dust particles, the presence of grains with $a_{\text {grain }} \sim \lambda / 2 \pi$ is required (for our observations this means $a_{\text {grain }} \gtrsim 250 \mu \mathrm{m}$ ). This is because the scattering cross-sections are too small unless the grains are made large. However, if the grains are too large, $a_{\text {grain }} \gtrsim 500 \mu \mathrm{m}$ for observations at $1 \mathrm{~mm}$, the polarisation efficiency becomes too small and polarised light is not efficiently produced (see, e.g. Kataoka et al. 2015). Scattering of radiation from a point source due to randomly aligned dust particles in an optically thin medium produces polarisation vectors tangential to the radiation field. Hence, in this scenario, our observations of non-tangential polarisation vectors would imply that the source of millimeter radiation is more complex, that the grains have a preferable alignment direction, or that the photons suffer multiple scattering episodes. We disfavour multiple scattering as a possible explanation because the effect of high scattering optical depths is to decrease the polarisation degree, as shown by Kataoka et al. (2015). Their models show that a maximum polarisation degree of $2.5 \%$ is obtained in the optically-thin regime and decreases for larger optical depths. Regarding the asymmetry of the incident radiation field, the source of millimeter flux is clearly not point-like, with detectable emission arising from the star itself and from an extended N-NE feature, apart from clump $\mathrm{C}$. The direction of the polarisation vectors cannot be explained by the $\mathrm{N}-\mathrm{NE}$ feature contributing to the source of unpolarized photons, because that would cause the vectors to be rotated in the opposite direction we observe. If we consider photons emitted by clump C itself to be the primary source of unpolarized radiation, the resolution of the observations would cause any polarisation created to be erased, unless clump $\mathrm{C}$ has a very special morphology. We consider this explanation unlikely, as higher-angular-resolution observations indicate that Clump C is elongated but still approximately spherically symmetric $(\mathrm{OG}+15)$. Moreover, none of these possible solutions is able to explain the direction of the polarisation vectors in visible wavelengths naturally.

The polarisation at millimeter wavelengths can also be explained by emission of dust particles aligned with the local magnetic field. Several alignment mechanisms have been proposed (For recent reviews, see e.g. Voshchinnikov 2012; Andersson et al. 2015). The currently favoured theory for this alignment is the Radiative Alignment Torque (RAT) theory (e.g. Lazarian \& Hoang 2007). In this case, the grains are expected to spin about their minor axis, with their major axis aligned perpendicularly to the magnetic field lines. Hence, the direction of polarisation of photons emitted by the dust would be perpendicular to that of the magnetic field lines. This scenario can also explain the direction of the polarisation seen in visible wavelengths, because absorption and scattering rather than emission are expected to dominate at visible wavelengths. Therefore, background scattered visible light would be more efficiently absorbed or scattered out of the line-of-sight in the direction of grain alignment. The resulting polarisation would then have a direction parallel to that of the magnetic field lines and perpendicular to the polarisation observed in emission. This is exactly what we find in our observations.

The RAT theory was mainly developed for interstellar dust grains. To investigate if the mechanism would work in the much denser circumstellar environment we need to compare the alignment timescale, given by the Larmor precession timescale, with the timescale set by gas-dust interaction (e.g. Hoang \& Lazarian 2009; Reissl et al. 2016). This comparison yields a lower limit for the magnetic field strength that, following Hoang \& Lazarian (2009), can be written as:

$|B|>4.1 \times 10^{-11} \frac{a}{s^{2}} n_{\mathrm{g}} T_{\mathrm{d}} \sqrt{T_{\mathrm{g}}}[\mathrm{G}]$.

We assume typical grains with a grain size of $a=10^{-5} \mathrm{~cm}$ $[=0.1 \mu \mathrm{m}]$ and aspect ratio $s=0.5$. We now assume the gas and dust temperature to be equal to the maximum allowed by the observations of clump C, $T_{\mathrm{d}}=T_{\mathrm{g}}=100 \mathrm{~K}$. The gas particle density $n_{\mathrm{g}}$ is more uncertain, as no molecular gas has yet been detected in clump C (e.g. OG+15, De Beck et al. 2015). Taking the dust mass derived from our measured fluxes, the size of clump $\mathrm{C}$ from $\mathrm{OG}+15$, and assuming a gas-to-dust ratio of 100 , we find $n_{\mathrm{g}} \approx 9 \times 10^{9} \mathrm{~cm}^{-3}$. For the dust alignment to be efficient, we find $B>13 \mathrm{mG}$ (or less if clump $\mathrm{C}$ is indeed severely depleted in gas). This increases to $\sim 65 \mathrm{mG}$ for grain sizes of $0.5 \mu \mathrm{m}$ that are suggested to exist in part of the VY CMa outflow (Scicluna et al. 2015). Even taking into account uncertainties on grain size and aspect ratios, and gas temperature and density, these values are still less than the field strength $(>100 \mathrm{mG})$ derived from $\mathrm{H}_{2} \mathrm{O}$ masers at a similar distance to the star as clump C (Vlemmings et al. 2002). Even in the strong radiation field of VY CMa, the radiative alignment timescale, following Hoang \& Lazarian (2009), becomes of the order of a few years. This is still significantly more than the Larmor precession timescale for a $13 \mathrm{mG}$ field of only $\sim 0.5$ day. This means that the grains will have had the time to align with the magnetic fields. Emission from aligned grains under the RAT alignment theory can thus explain our observations. 
In addition to the RAT alignment theory, a number of other grain alignment theories exist. Several of these theories make specific predictions and so far only the RAT theory can explain all observational characteristics of aligned dust (e.g. Voshchinnikov 2012; Andersson et al. 2015). However, different mechanisms can operate or dominate in different astrophysical regions, and the special conditions in the dense circumstellar environment can provide further tests for the different alignment mechanisms. The earliest proposed mechanism is the imperfect Davis-Greenstein (IDG) mechanism (Davis \& Greenstein 1951) that has seen several adjustments over the last decades. In this mechanism the dissipation of magnetisation energy (for grains including small amounts of iron) aligns the spin axis with the magnetic field. This theory specifically aligns the small grains and the alignment efficiency depends on the temperature difference between the dust and the gas. Under our earlier assumptions, of equal dust- and gas-temperatures, we would thus not expect any alignment. However, further detailed observations of clump $\mathrm{C}$ are needed to determine if conditions are such that the IDG mechanism can operate. Thus, while our observations are fully consistent with the RAT theory, and provide strong constraints on the alignment timescales, we cannot yet rule out other mechanisms that align the grains with the magnetic field.

\subsection{Magnetic field morphology}

Having determined that the linear polarisation of the majority of the $\mathrm{SiO}$ masers traces the magnetic field, we notice, with the exception of the potentially saturated maser feature at $V_{\text {lsr }}=$ $15 \mathrm{~km} \mathrm{~s}^{-1}$, a clear structure in the $v=1{ }^{28} \mathrm{SiO}$ EVPAs. The vectors rotate from $\sim 130^{\circ}$ at blue-shifted velocities to $\sim-50^{\circ}$ around the stellar velocity and back to $\sim 140^{\circ}$ on the red-shifted side. Almost exactly the same rotating behaviour was seen in the $86 \mathrm{GHz}{ }^{28} \mathrm{SiO} J=2-1, v=1$ polarisation vectors observed by Herpin et al. (2006). The EVPAs we observe are also consistent with the angles measured for the ${ }^{28} \mathrm{SiO}(J=5-4, v=1)$ masers in Shinnaga et al. (2004).

The actual magnetic field direction is parallel or perpendicular to the measured polarisation vectors, depending on whether the angle $\theta$ between the maser propagation and the line of sight is respectively smaller or larger than the Van Vleck angle $\theta_{\mathrm{cr}} \approx 55^{\circ}$, where the fractional polarisation is equal to zero. Considering the dust polarisation vectors arising in clump $\mathrm{C}$, which is thought to be close to the plane of the stellar velocity, are perpendicular to the magnetic field direction, the same appears to be true for the $\mathrm{SiO}$ masers. For most masers, we can thus conclude that $\theta>55^{\circ}$ and that the magnetic field is perpendicular to the $\mathrm{SiO}$ maser EVPA. That is also consistent with the $\sim 40^{\circ}$ magnetic field angle determined from the extreme red-shifted thermal SiO.

Such polarisation behaviour may be consistent with a predominantly toroidal field morphology, with an inclination $>55^{\circ}$ and a position angle on the sky of $\sim 40^{\circ}$. The magnetic field strength dependence on radius of $B \propto r^{-1}$ for a toroidal field also fits the field strength derived from the $\mathrm{SiO}$ masers observed here, as well as previous observations (Herpin et al. 2006; Richter et al. 2016), together with the $\mathrm{OH}$, and $\mathrm{H}_{2} \mathrm{O}$ masers (Vlemmings et al. 2002).

The hypothesis of a predominantly toroidal magnetic field, however, can only be confirmed with high-angular-resolution observations, as it depends on the position of the individual maser components. Component fitting of the $\mathrm{SiO}$ masers presented here indicates that they occur within $\sim 20$ mas, or $\sim 2 R_{*}$, from the star. As the positional uncertainty for most masers is of similar order, it is not possible to produce a more detailed map of the magnetic field with the current data.

Assuming a toroidal field configuration would place clump C in the magnetic equator, possibly the result of a magnetically controlled ejecta. This scenario is supported by the observations of Richter et al. (2016). Although on much smaller scales, their VLBI observations indicate elongation of $v=1, J=1-0$ $\mathrm{SiO}$ maser features from the star in the general direction of clump C. For these masers they derive a 2-5 Gauss magnetic field strength. Richter et al. (2016) speculate that the elongated features, at least their "F1" feature studied in detail, may be associated with a highly magnetized outflow above a stellar surface magnetic cool spot or convective cell.

\section{Conclusions}

The ALMA Band $5178 \mathrm{GHz}$ continuum observations of VY CMa reveal that the dust structure at this wavelength is similar to the structure found in previous 321, and $658 \mathrm{GHz}$ ALMA observations. The derived spectral index map indicates the dust of clump $\mathrm{C}$ is mostly optically thick even at $178 \mathrm{GHz}$. Assuming standard dust properties, this means the estimated clump $\mathrm{C}$ dust mass is $>1.2 \times 10^{-3} M_{\odot}$.

Significant linear polarisation of up to $3 \%$ is detected from clump C. This is the first detection of dust polarisation at millimeter wavelengths in the inner circumstellar environment of an evolved star. The EVPA is $\sim 8^{\circ}$ and is neither radial nor tangential to the stellar radiation field. If the continuum polarisation is the result of emission from magnetically aligned dust grains, then the observations indicate that, according to RAT grain alignment theory, the grains cannot be diamagnetic (e.g. purely carbonaceous). This is not unexpected for the oxygen rich environment of VY CMa where for example $\mathrm{Si}, \mathrm{Mg}$, and/or Fe are expected to be present in the dust. The magnetic field would have a preferred angle of $98^{\circ}$ and should be $>13 \mathrm{mG}$ in strength. As higher field strengths of 0.1 to a few Gauss are measured in the maser region, the magnetic field thus has no problem aligning the dust grains in clump C. Higher angular resolution observations could subsequently be done to determine the magnetic field structure and its possible role in confining the dense clump C. Although we show it to be unlikely, we cannot yet completely rule out a self-scattering origin of the polarisation. In this case, the grains in clump $\mathrm{C}$ would need to be extremely large $(>250 \mu \mathrm{m})$. The origin of the preferred polarisation direction would then also imply a very specific dust geometry.

The linear polarisation of the $\mathrm{SiO}$ masers is typically $\sim 5-$ $10 \%$, and goes up to $\sim 20-30 \%$ for specific features. The average polarisation is at the level that could be expected from regular maser polarisation theory of non- to intermediately saturated masers, and does not require anisotropic pumping of the magnetic substates. The EVPA is also neither tangential nor radial and indicates a possible toroidal magnetic field morphology. For the thermal $\mathrm{SiO}$, the linear polarisation appears consistent with a direction set by the stellar radiation field at the blueshifted side, but not at the redshifted side, where it also seems to probe the magnetic field.

Circular polarisation is also detected for the maser and thermal lines. The magnitude and spectral behaviour of the circular polarisation indicates that it is unlikely to be caused by standard instrumental effects or calibration errors. The observed polarisation is also consistent with previous measurements. Finally, since the calibrators and VY CMa continuum show no sign of circular polarisation and the Stokes $V$ characteristics are very 
different for the maser and thermal lines, we suggest that the circular polarisation is likely intrinsic to the source. However, as this constitutes the first circular polarisation imaged with ALMA, additional observations are needed to rule out possible unknown instrumental contributions to Stokes $V$ and confirm our observations. A normal Zeeman interpretation of the maser lines would imply a field of $\sim 1-3 \mathrm{G}$ in the $\mathrm{SiO}$ maser region of VY CMa. This is consistent with previous lower frequency $\mathrm{SiO}$ and $\mathrm{H}_{2} \mathrm{O}$ maser observations and the toroidal field interpretation. The circular polarisation of the thermal line could potentially originate from resonant scattering of magnetized $\mathrm{SiO}$ gas at a greater distance from the star.

Acknowledgements. This work was supported by ERC consolidator grant 614264. W.V. and T.K. also acknowledge support from the Swedish Research Council. This paper makes use of the following ALMA data: ADS/JAO.ALMA\#2011.0.00011.S. ALMA is a partnership of ESO (representing its member states), NSF (USA) and NINS (Japan), together with NRC (Canada), NSC and ASIAA (Taiwan), and KASI (Republic of Korea), in cooperation with the Republic of Chile. The Joint ALMA Observatory is operated by ESO, AUI/NRAO and NAOJ. We thank the ALMA Band 5 Science Verification team for their efforts in preparing and performing the observations and the ARC node network reduction team and the hosts of the Nordic ARC for their reduction work.

\section{References}

Andersson, B.-G., Lazarian, A., \& Vaillancourt, J. E. 2015, ARA\&A, 53, 501 Cortes, P. C., Girart, J. M., Hull, C. L. H., et al. 2016, ApJ, 825, L15 Danchi, W. C., Bester, M., Degiacomi, C. G., Greenhill, L. J., \& Townes, C. H. 1994, AJ, 107, 1469

Davis, L., Jr., \& Greenstein, J. L. 1951, ApJ, 114, 206

De Beck, E., Vlemmings, W., Muller, S., et al. 2015, A\&A, 580, A36

Decin, L., Hony, S., de Koter, A., et al. 2006, A\&A, 456, 549

Elitzur, M. 1996, ApJ, 457, 415

Etoka, S., \& Diamond, P. J. 2010, MNRAS, 406, 2218

Girart, J. M., Patel, N., Vlemmings, W. H. T., \& Rao, R. 2012, ApJ, 751, L20

Glenn, J., Walker, C. K., Bieging, J. H., \& Jewell, P. R. 1997, ApJ, 487, L89

Goldreich, P., \& Kylafis, N. D. 1981, ApJ, 243, L75

Goldreich, P., \& Kylafis, N. D. 1982, ApJ, 253, 606

Habing, H. J. 1996, A\&ARv, 7, 97
Hartmann, L., \& MacGregor, K. B. 1980, ApJ, 242, 260

Herpin, F., Baudry, A., Thum, C., Morris, D., \& Wiesemeyer, H. 2006, A\&A, 450,667

Hoang, T., \& Lazarian, A. 2009, ApJ, 697, 1316

Höfner, S. 2008, A\&A, 491, L1

Houde, M. 2014, ApJ, 795, 27

Humphreys, E. M. L., Immer, K., Gray, M. D., et al. 2017, A\&A, 603, A77

Humphreys, R. M., Helton, L. A., \& Jones, T. J. 2007, AJ, 133, 2716

Jones, T. J., Humphreys, R. M., Helton, L. A., Gui, C., \& Huang, X. 2007, AJ, 133,2730

Josselin, E., \& Plez, B. 2007, A\&A, 469, 671

Kataoka, A., Muto, T., Momose, M., et al. 2015, ApJ, 809, 78

Kemball, A. J., \& Diamond, P. J. 1997, ApJ, 481, L111

Kylafis, N. D. 1983, ApJ, 267, 137

Lazarian, A., \& Hoang, T. 2007, MNRAS, 378, 910

Morris, M., Lucas, R., \& Omont, A. 1985, A\&A, 142, 107

Martí-Vidal, I., Vlemmings, W. H. T., Carozzi, T., et al. 2016, ALMA Band 5 VY CMa Science Verification data release memo

Müller, H. S. P., Thorwirth, S., Roth, D. A., \& Winnewisser, G. 2001, A\&A, 370, L49

Nedoluha, G. E., \& Watson, W. D. 1990, ApJ, 354, 660

Norris, B. R. M., Tuthill, P. G., Ireland, M. J., et al. 2012, Nature, 484, 220

O'Gorman, E., Vlemmings, W., Richards, A. M. S., et al. 2015, A\&A, 573, L1

Pérez-Sánchez, A. F., \& Vlemmings, W. H. T. 2013, A\&A, 551, A15

Reissl, S., Wolf, S., \& Brauer, R. 2016, A\&A, 593, A87

Richards, A. M. S., Impellizzeri, C. M. V., Humphreys, E. M., et al. 2014, A\&A, 572, L9

Richter, L., Kemball, A., \& Jonas, J. 2016, MNRAS, 461, 2309

Sabin, L., Zhang, Q., Zijlstra, A. A., et al. 2014, MNRAS, 438, 1794

Scicluna, P., Siebenmorgen, R., Wesson, R., et al. 2015, A\&A, 584, L10

Shinnaga, H., Moran, J. M., Young, K. H., \& Ho, P. T. P. 2004, ApJ, 616, L47

Speck, A. K., Barlow, M. J., Sylvester, R. J., \& Hofmeister, A. M. 2000, A\&AS, 146,437

Vlemmings, W. H. T., Diamond, P. J., \& van Langevelde, H. J. 2002, A\&A, 394, 589

Vlemmings, W. H. T., van Langevelde, H. J., \& Diamond, P. J. 2005, A\&A, 434, 1029

Vlemmings, W. H. T., Ramstedt, S., Rao, R., \& Maercker, M. 2012, A\&A, 540, L3

Voshchinnikov, N. V. 2012, J. Quant. Spectr. Rad. Transf., 113, 2334

Watson, W. D. 2002, in Cosmic Masers: From Proto-Stars to Black Holes, eds. V. Migenes, \& M. J. Reid, IAU Symp., 206, 464

Woitke, P. 2006, A\&A, 460, L9

Zhang, B., Reid, M. J., Menten, K. M., \& Zheng, X. W. 2012, ApJ, 744, 23 


\section{Appendix A: Monte Carlo analysis of spurious circular polarisation}

The calibration of the instrumental polarisation can be described by two different quantities: on the one hand, the relative phase between the polarisers at the reference antenna (we call this quantity $X-Y$ phase) and, on the other hand, the leakage between the polarisers of all the antennas, which are modelled by complex-valued quantities called D-terms. In order to simulate the effects that a residual $X-Y$ phase and/or polarisation leakage would have on the final $\mathrm{SiO}$ images in Stokes $V$, we have performed a Monte Carlo analysis. We have assumed that both the $X-Y$ phase and the D-terms are smooth functions of the frequency within the band, so that the channel noise in the calibration plots shown in (Martí-Vidal et al. 2016) reflects the uncertainties related to the process of antenna-gain estimation. All these uncertainties combined may bias the final images, introducing spurious signals in all four Stokes parameters.

Judging from the calibration plots in (Martí-Vidal et al. 2016, their Fig. 8), we estimate an upper bound in the $X-Y$ phase uncertainty of $\sim 10$ degrees. Regarding the D-terms (Fig. 11), the typical scatter among neighbouring frequency channels (of the same antenna) is $1-2 \%$.

Based on these assumptions, we have generated one thousand sets of random D-terms for all the ALMA antennas, together with random $X-Y$ phases for the reference antenna. Then, we applied these quantities to the calibrated data and generated modified images of VY CMa for all four Stokes parameters. The random D-terms in our analysis follow a Gaussian distribution with a $1 \sigma$ amplitude of $1 \%$. The $X-Y$ phase follows a Gaussian distribution with a $1 \sigma$ deviation of 10 degrees.
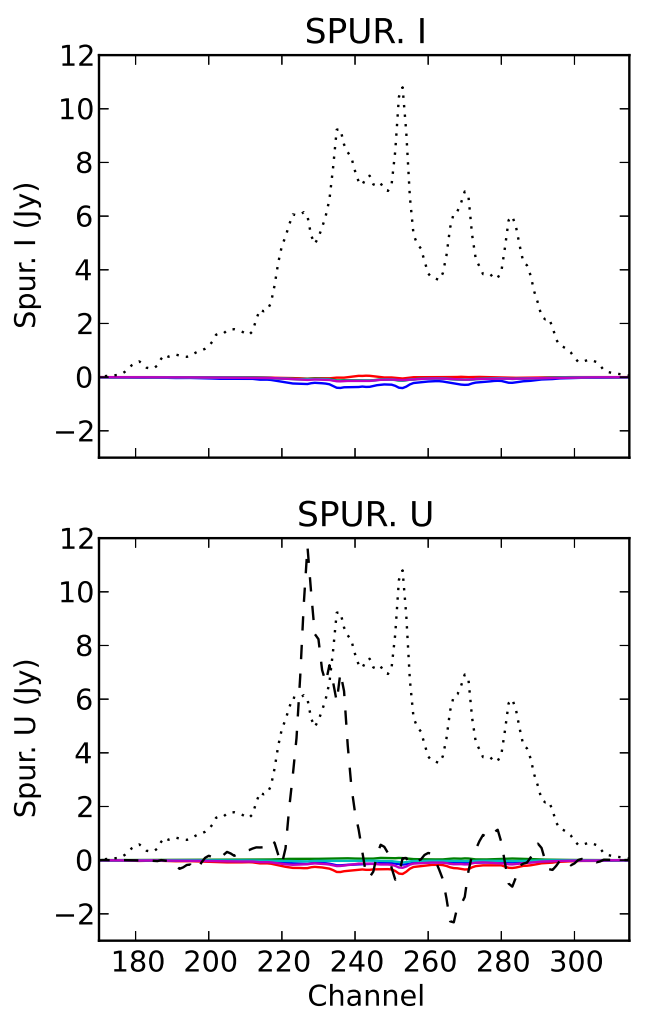

After the imaging, we have computed the difference between the calibration-corrupted full-polarisation images and those obtained from the original calibration (we call this difference the spurious source polarisation). In Figs. A.1, and A.2, we show the spurious polarisation (in all four Stokes parameters) resulting from five representative Monte Carlo iterations for spw 3 and 4. The spectra are taken at the peak intensity of Stokes $I$.

A seen in Fig. A.1, we notice that the spurious fractional Stokes $V$ has typical wide-band average values of the order of $\pm 0.1 \%$ across the entire $\mathrm{SiO}$ line. This is similar to the measured average Stokes $V$ from the original polarisation calibration: $-0.1 \%$ and indicates our assumed uncertainties in the calibration are correct.

In any case, the spurious Stokes $V$ in our Monte Carlo analysis is typically below $0.1 \%$ in absolute value, and has a smooth frequency dependence across the line. On the contrary, the Stokes $V$ recovered from the original calibration shows a much stronger frequency dependence, with extreme changes between close by channels that are up to 4-5 times higher than those seen in the spurious Stokes $V$. This is a clear indication that the differential Stokes $V$ (i.e. the frequency dependence of Stokes $V$ ) is a robust quantity, almost independent of calibration biases. A similar conclusion can be derived from the Monte Carlo analysis on spw 4 (Fig. A.2). We can thus conclude that the observed Stokes $V$ behaviour of the $\mathrm{SiO}$ lines is likely real and not an artifact of calibration. However, to rule out an unknown instrumental effect introduced in the ALMA system would require further observations.
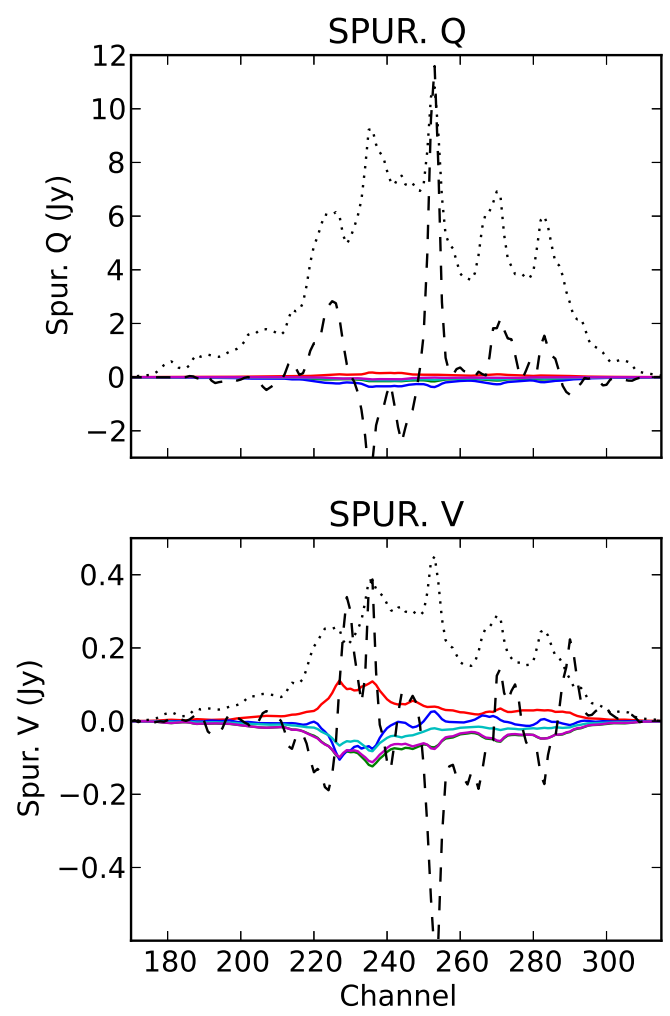

Fig. A.1. Spurious polarisation in spw 3 (solid lines) for five representative Monte Carlo simulations. The spectra are extracted at the position of the line peak. The quantities are given in Jy. The short dashed line indicates a total intensity spectrum (not to scale) for illustration. The long-dashed lines are the observed $Q, U$, and $V$ polarisations at the same scale as the simulated spurious polarisation spectra. It is clear that the magnitude and spectral behaviour of the observed spectra is significantly different from the spurious signal. 

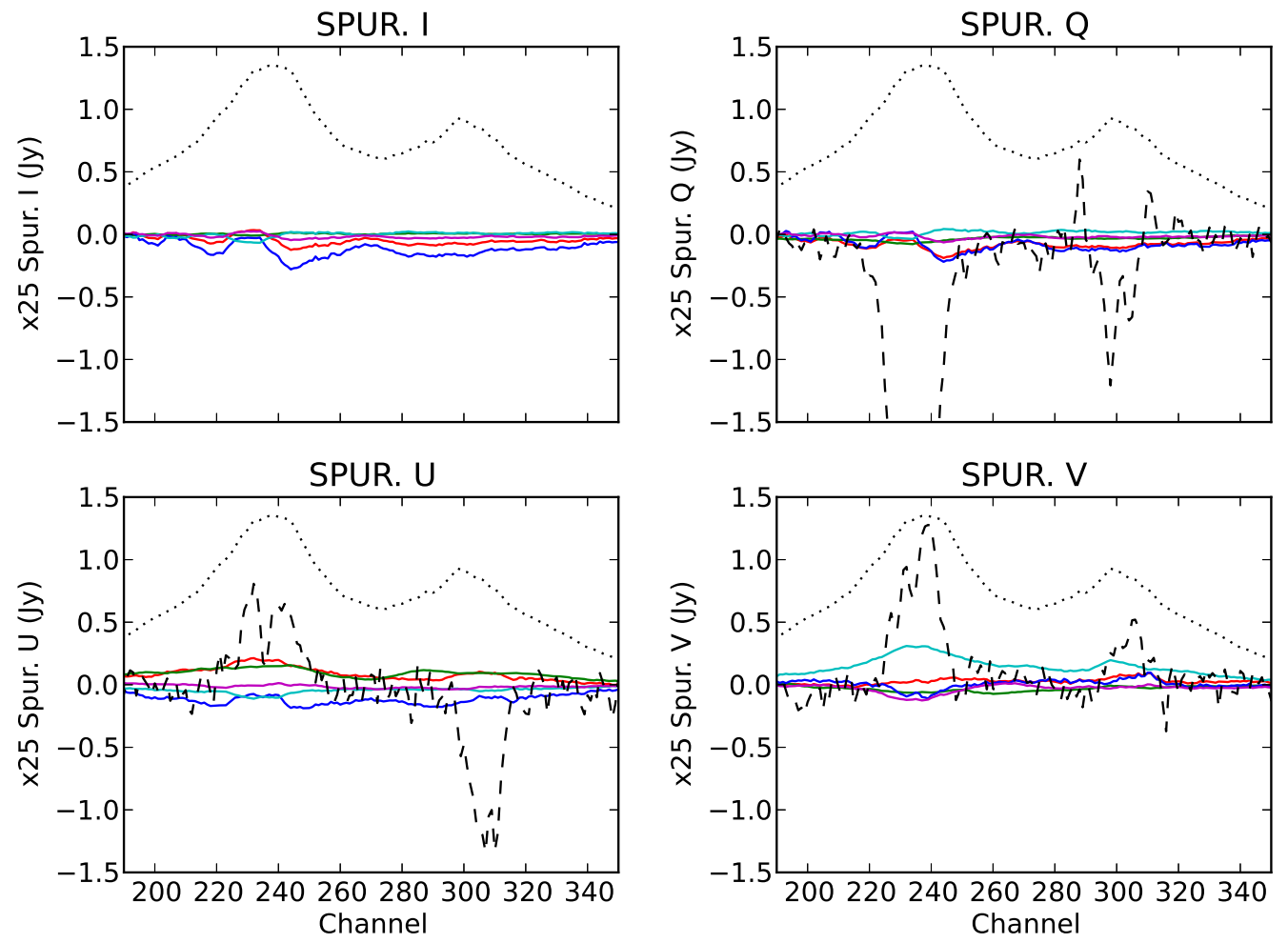

Fig. A.2. Same as Fig. A.1, but for spw 4. We note that the spectra are multiplied by 25 . 